\title{
Editorial
}

\section{Raman Spectroscopy of Crystals}

\author{
Alexander Krylov(i) \\ Kirensky Institute of Physics, Federal Research Center KSC SB RAS, 660036 Krasnoyarsk, Russia; \\ shusy@iph.krasn.ru
}

Received: 26 October 2020; Accepted: 27 October 2020; Published: 29 October 2020

check for updates

Raman spectroscopy is now finding wide-ranging application in pure and applied science. It can be used for the characterization of the atomic structure of solids because Raman scattering depends on the polarisation and direction of the incident light, the crystal symmetry and orientation of the solid sample, and the direction and polarisation of the scattered light. Raman spectroscopy can play a significant role in the study of crystal phase transformations, and it could involve order-disorder phenomena, polymorphs, chemical diffusion, and solid-solution shifts. One can study crystals in situ in extreme conditions - under high pressure, at high temperatures or both simultaneously, at low temperatures, or in electric or magnetic fields and extract thermodynamic data from the variations in the Raman bands with varying pressure-temperature-field conditions. Other aspects that merit attention are the possibility of analyzing nanocrystals, crystalline microinclusions under the surface, organic crystals and many others.

Once one is armed with sufficient understanding about Raman spectroscopy applied to crystals, one may then begin to study other research domains where crystals constitute the raw material on which everything else depends. In some cases, like optics or electronics or gemmology, the fundamental object of interest is still an individual monocrystal. However, in most cases, studies concern an association, of crystals, which, by definition, constitutes a rock in the case of natural minerals, and the appropriate discipline is geology. Along with systematic mineralogy, geology belongs to the entire Earth sciences, to which one often adds extraterrestrial materials like meteorites as well as biominerals. Where the crystals are not natural, this concerns synthetic materials of relevance to the material sciences involving chemistry and physics and much of industry, like ceramics, semiconductors or superconductors. In yet other domains, the crystals are accompanied by fluids, whether gaseous or liquid, notably of organic origin in the domain of life sciences and yet other industries like medicine, agricultural or pharmaceutical.

This review briefly summarises papers published recently in the Special Issue "Raman Spectroscopy of Crystals". It is hoped that this review will provide an up-to-date view of the field of Raman spectroscopy in crystallin matter and will be useful to those who evolve Raman technique in general or use it as a spectroscopic tool for scientific application.

The effect of the compositional characteristics, charge technology, and crystals on the Raman spectrum of real $\mathrm{LiNbO}_{3}$ crystals grown by Czochralski was reviewed by Sidorov et al. [1]. Their review focuses on the interpretation of the processes of the Raman scattering in nominally pure and doped LN crystals with structural defects which were formed during growth of LN crystals of different chemical composition. Conoscopic patterns of the studied LN crystals indicate their high compositional uniformity. Authors reported the intermittent structure of the laser beam in nominally pure non-gyrotropic crystal. This structure may be due to the formation of bound states of two photons as a result of various nonlinear-optical processes: two-photon absorption, the stimulated Raman scattering. In the $\mathrm{Zn}$-doped Raman spectra of $\mathrm{LiNbO}_{3}$, crystals revealed a region of increased structure ordering when the order of alternation of the main, doping cations and vacancies along the polar axis is increased in the cation sublattice, and oxygen octahedra are almost perfect.

The Raman spectroscopy techniques and their use in structural biology, biophysics, cells, and tissues imaging towards the development of various medical diagnostic tools, drug design, 
and other medical applications were reviewed by Alexey V. Vlasov et al. Classical and contemporary structural studies of different water-soluble and membrane proteins, DNA, RNA, and their interactions and behaviour in different systems were analyzed in terms of applicability of Raman spectroscopy and their complementarity to other corresponding methods. The Raman spectroscopy links the fundamental structural biology and its medical applications in cancer, cardiovascular, neurodegenerative, atherosclerotic, and other diseases. The key roles of this technique in modern technologies of structure-based drug design are the detection and imaging of membrane protein microcrystals with the help of coherent anti-Stokes Raman scattering, which could help to further the development of protein structural crystallography and would result in many novel high-resolution structures of membrane proteins-drug targets; and, structural studies of photoactive membrane proteins (rhodopsins, photoreceptors and others) for the development of new optogenetic tools. Physical background and biomedical applications of spontaneous, stimulated, resonant, and surfaceand tip-enhanced Raman spectroscopy are also discussed [2].

The in-depth analysis of excitation-dependent resonance Raman spectroscopy at a sub-band-gap excitation of GaN:(Mn, Mg) with Mn-Mgk IR-emitting cation complexes completed by Andrii Nikolenko et al. In addition, a resonance Raman analysis at the inter-band excitation energy is presented in order to estimate the strength of the electron-phonon interaction from the relative intensity of the 2LO and 1LO Raman resonances [3].

Hua-Yi Hou et al. presented a review of anomalous behaviours of spin waves observed by inelastic light scattering experiments, especially by Raman spectroscopy. The large asymmetry of anti-Stokes to Stokes intensity ratio, broad linewidth, strong resonance effect, unique polarisation selection, and abnormal impurity dependence of spin waves are discussed, and a proposed model for the mechanisms of a spin flip, spin relaxing, and spin-wave scattering is presented for understanding these anomalous behaviours of spin waves. Besides, two magnonic crystal structures are proposed for manipulating spin waves through analyses of the abnormal impurity dependence of spin waves. The review of the anomalous behaviours of spin waves and the proposed models provide the directions for an easier experimental study of spin waves by inelastic light scattering, which will be useful for raising the research efforts for investigating spin waves [4]

Irena Nemtsov et al. presented a new method for the investigation of crystal structures by studying vibrational modes obtained from each face of a single crystal using low-wavenumber Raman spectroscopy. This method does not require any transparency of the measured crystal, as collecting Raman scattering is done in reflection mode. Moreover, the crystal diameter can be as small as the focus of the exciting laser, where a low-wavenumber Raman was employed to study the correlation between the signal intensity at three incident beam directions and the orientations of intermolecular interactions when excited from multiple faces [5].

The Raman depth profiling to determine the different Raman properties from different layers of $4 \mathrm{H}-\mathrm{S} 1 \mathrm{iC}$ samples with a multilayer structure was successfully applied by Tao Liu et al. Raman scattering measurements with exciting photons having an energy above the band gap of $4 \mathrm{H}-\mathrm{SiC}$ can be used to determine carrier concentrations of rather lowly doped samples. The proposed approach is based upon a linear relationship between the effective free carrier concentration and the laser power from which the carrier concentration of the n-type epitaxial layer. This could extend the lowest measurable carrier concentration [6].

Yanshan Yin et al. reported the use of FTIR, Raman spectroscopy, and XRD as complementary techniques for the identification of the minerals in coals and coal ashes. The Raman spectroscopy indicated the occurrence of anatase, quartz, anhydrite, and hematite in the coal ashes. The infrared spectra of raw coals demonstrate intense absorption bands of kaolinite and weak bands of organic functional groups because of the relatively high silicates content of the coals. The second-derivative infrared spectra clearly showed separated individual bands and thus present detailed mineral components of the coals and coal ashes. Comparison of the infrared spectra of coals and coal ashes demonstrated the thermal transformation of minerals such as kaolinite, calcite, and gypsum. 
Raman spectra of raw coals showed intense carbon peaks but no mineral matter peaks. The XRD analyses of mineral composition of coals and coal ashes generally agreed with the results of infrared and Raman spectra [7].

The torsional vibrational modes behaviour investigation of the ammonium group from L-valine, L-isoleucine, taurine, and L-arginine hydrochloride monohydrate submitted to temperature variation was reported by André Luís de Oliveira Cavaignac et al. A comparative study was carried out among these four monocrystals related to the behaviour of the vibrational modes provides insights about the hydrogen bond changes. The factors' influence on the system stability was also discussed [8].

Xiuyan Chen et al. reports the results of the Raman spectrum investigations of $\alpha$-quartz and $\beta$-quartz crystals were simulated in the Material Studio software under different levels of temperature and pressure. On the model basis, $\mathrm{Pb}^{4+}$-doped $\alpha$-quartz and $\beta$-quartz crystals were constructed, and their Raman spectra were calculated. The analyzed effect of $\mathrm{Pb}^{4+}$ doping on $\mathrm{SiO}_{2}$ crystal's Raman expands the quartz crystal's application, provided a new crystal type, as well the demonstration of the Raman frequency shift as the function of $\mathrm{Pb}^{4+}$ [9].

Finally, the rigorous analysis of the temperature behaviour of the Raman spectra of diphenylalanine microtubes was reported by Alexander Krylov et al. Authors found several temperature-dependent spectral anomalies. The spectra after passing through the critical temperature change significantly and are not restored upon cooling. The observed transition is thus irreversible. The mechanism and processes occurring at the phase transition are related to rather small changes in diphenylalanine structure, causing the modifications of the phenyl-phenyl interaction region in Raman spectra. The evaporation of water at critical temperature leads to significant and irreversible changes in the crystal lattice. The appearance of new lines in the $\mathrm{C}-\mathrm{H}$ stretching region was associated with the previously observed cyclization of diphenylalanine molecules. The temperature behaviour of the spectral lines in the region of lattice vibrations indicates the changes in diphenylalanine microtubes at several different temperatures. These changes are concentrated in the area of vibration of certain molecular groups. Some anomalies were associated with the water state changes inside microtubes [10].

Funding: This research received no external funding.

Conflicts of Interest: The author declares no conflict of interest.

\section{References}

1. Sidorov, N.; Palatnikov, M.; Kadetova, A. Raman Scattering in Non-Stoichiometric Lithium Niobate Crystals with a Low Photorefractive Effect. Crystals 2019, 9, 535. [CrossRef]

2. Vlasov, A.V.; Maliar, N.L.; Bazhenov, S.V.; Nikelshparg, E.I.; Brazhe, N.A.; Vlasova, A.D.; Osipov, S.D.; Sudarev, V.V.; Ryzhykau, Y.L.; Bogorodskiy, A.O.; et al. Raman Scattering: From Structural Biology to Medical Applications. Crystals 2020, 10, 38. [CrossRef]

3. Nikolenko, A.; Strelchuk, V.; Tsykaniuk, B.; Kysylychyn, D.; Capuzzo, G.; Bonanni, A. Resonance Raman Spectroscopy of $\mathrm{Mn}_{-\mathrm{Mg}_{\mathrm{k}}}$ Cation Complexes in GaN. Crystals 2019, 9, 235. [CrossRef]

4. Hou, H.-Y.; Yang, M.; Qiu, J.; Yang, I.-S.; Chen, X.-B. Anomalous Behaviors of Spin Waves Studied by Inelastic Light Scattering. Crystals 2019, 9, 357. [CrossRef]

5. Nemtsov, I.; Aviv, H.; Mastai, Y.; Tischler, Y.R. Polarization Dependence of Low-Frequency Vibrations from Multiple Faces in an Organic Single Crystal. Crystals 2019, 9, 425. [CrossRef]

6. Liu, T.; Xu, Z.; Rommel, M.; Wang, H.; Song, Y.; Wang, Y.; Fang, F. Raman Characterization of Carrier Concentrations of Al-implanted $4 \mathrm{H}-\mathrm{SiC}$ with Low Carrier Concentration by Photo-Generated Carrier Effect. Crystals 2019, 9, 428. [CrossRef]

7. Yin, Y.; Yin, H.; Wu, Z.; Qi, C.; Tian, H.; Zhang, W.; Hu, Z.; Feng, L. Characterization of Coals and Coal Ashes with High Si Content Using Combined Second-Derivative Infrared Spectroscopy and Raman Spectroscopy. Crystals 2019, 9, 513. [CrossRef]

8. Cavaignac, A.L.O.; Lima, R.J.C.; Freire, P.T.C. The Behavior of $\mathrm{NH}_{3}^{+}$Torsional Vibration in Amino Acids: A Raman Spectroscopic Study. Crystals 2019, 9, 517. [CrossRef] 
9. Chen, X.; Feng, W.; Zhang, G.; Gao, Y. Raman Spectra of Quartz and $\mathrm{Pb}^{4+}$-Doped $\mathrm{SiO}_{2}$ Crystals at Different Temperature and Pressure. Crystals 2019, 9, 569. [CrossRef]

10. Krylov, A.; Krylova, S.; Kopyl, S.; Krylov, A.; Salehli, F.; Zelenovskiy, P.; Vtyurin, A.; Kholkin, A. Raman Spectra of Diphenylalanine Microtubes: Polarisation and Temperature Effects. Crystals 2020, 10, 224. [CrossRef]

Publisher's Note: MDPI stays neutral with regard to jurisdictional claims in published maps and institutional affiliations.

(c) 2020 by the authors. Licensee MDPI, Basel, Switzerland. This article is an open access article distributed under the terms and conditions of the Creative Commons Attribution (CC BY) license (http:/ / creativecommons.org/licenses/by/4.0/). 